BULL. AUSTRAL. MATH. SOC.

$05 \mathrm{C} 25,20 \mathrm{~B} 2$ :

VOL. $7(1962), 131-134$.

\title{
An algebraic characterization of symmetric graphs with a prime number of vertices
}

\section{J.L. Berggren}

A graph $\Gamma$ is called symmetric if its automorphism group is transitive on its vertices and edges. Let $p$ be an odd prime, $Z(p)$ the field of integers modulo $p$, and $Z^{*}(p)=\{a \in Z(p) \mid a \neq 0\}$, the multiplicative subgroup of $2(p)$. This paper gives a simple proof of the equivalence of two statements :

(1) $\Gamma$ is a symmetric graph with $p$ vertices, each having degree $n \geq 1$;

(2) the integer $n$ is an even divisor of $p-1$ and $\Gamma$ is isomorphic to the graph whose vertices are the elements of $Z(p)$ and whose edges are the pairs $\{a, a+h\}$ where $a \in Z(p)$ and $h \in H$, the unique subgroup of $Z^{*}(p)$ of order $n$.

In addition, the automorphism group of $\Gamma$ is determined.

The results of this paper are not new, for they were conjectured in [5] and proved in [3]. However the proof given here is very much simpler than that of [3], for the author of that paper used results he proved in [2] by means of Schur's theory of simply-transitive permutation groups. The proof given in this paper, using methods similar to those in [1], shows the elementary nature of the main result of [3].

Received 22 March 1972. 
In this paper, $p$ will denote an odd prime, $Z(p)$ the field of integers modulo $p$, and $Z^{*}(p)$ the multiplicative subgroup of non-zero elements in $Z(p)$. Because $Z^{*}(p)$ is cyclic of order $p-1$ it has, for each divisor $n$ of $p-1$, a unique subgroup of order $n$.

A graph $\Gamma$ is called symmetric if its automorphism group $G$ is transitive on its vertices and edges. Notice that each vertex of a symmetric graph has the same degree. In [5] it was proved that, of the following two statements, (ii) implies (i):

(i) $\Gamma$ is a symmetric graph with $p$ vertices each having degree $n \geq 1$;

(ii) the integer $n$ is an even divisor of $p-1$ and $\Gamma$ is isomorphic to the graph whose vertices are the elements of $Z(p)$ and whose edges are the pairs $\{a, a+h\}$ where $a \in Z(p)$ and $h \in H$, the unique subgroup of $Z^{*}(p)$ of order $n$.

We now prove that (i) implies (ii).

Let $\Gamma$ be as in ( $i$ ) and $G$ its automorphism group. If $G$ is doubly-transitive on the vertices then $\Gamma$ is obviously the complete graph on $p$ vertices and hence isomorphic to the graph constructed as in (ii) with $H=Z^{*}(p)$. In this case $n=p-1$. If $G$ is not

doubly-transitive on the vertices then by Theorem 7.3 of [4] we may suppose the vertices of $\Gamma$ are the points of $Z(p)$ and that $G \leq\left\{T(a, b) \mid a \in Z^{*}(p), b \in Z(p)\right\}=S$, where $T(a, b)$ is the permutation of $Z(p)$ which maps $x$ to $a x+b$.

Since $G$ is transitive on the $p$ vertices, $p|| G \mid$ and, since $K=\{T(1, b) \mid b \in Z(p)\}$ is the subgroup of order $p$ in $S$, we conclude $G \geq K$. It is now easy to verify that $H=\left\{a \in Z^{*}(p) \mid T(a, 0) \in G\right\}$ is a subgroup of $Z^{*}(p)$ and that $G=\{T(a, b) \mid a \in H, b \in Z(p)\}$.

We can now easily finish the proof. For each $i, j \in Z(p)$ we know $T(1,-i-j) \in G$, so $\{i, j\}$ is an edge of $\Gamma$ if and only if $\{-i,-j\}$ is an edge. Thus $T(-1,0) \in G$, so $-1 \in H$, and $H$ has even order. If $A(0)$ is the set of points joined to 0 by an edge then $A(0)=H c_{1}+\ldots+H c_{r}$, for the stabilizer of 0 is $\{T(a, 0) \mid a \in H\}$. If $f \geq 2$ then there is $T(a, b) \in G$ so that $T(a, b)$ maps 0 to $c_{2}$ 
and $c_{1}$ to 0 . This implies $b=c_{2}$ and $a c_{1}=-c_{2}$, that is, $(-a) c_{1}=c_{2}$. But, $(-1)$ and $a$ are in $H$ so $-a \in H$ and $H c_{1}=H c_{2}$. This contradiction shows $r=1$ so $n=|H|$, an even divisor of $p-1$. We have seen the edges of $\Gamma$ are the pairs $\left\{a, a+h c_{1}\right\}$, so the map $a \rightarrow a c_{1}^{-1}$ is an isomorphism from $\Gamma$ onto the graph $\Gamma^{\prime}$ whose vertices are the points of $Z(p)$ and whose edges are the pairs $\{a, a+h\}, a \in Z(p)$ and $h \in H$. Also if $G^{\prime}$ is the automorphism group of $\Gamma^{\prime}$ then $G^{\prime}=G$. For it is clear that $G^{\prime} \geq G$ and, since $\Gamma$ and $\Gamma^{\prime}$ are isomorphic, $G^{\prime}$ and $G$ are isomorphic.

In conclusion, we have shown that any symmetric graph $\Gamma$ with $p$ vertices has even degree $n$. Further, $n \mid(p-1)$ and if $H$ is the unique subgroup of $Z^{*}(p)$ of order $n$ then $\Gamma$ is isomorphic to the graph constructed from $H$ as in (ii). Also, if $H \neq Z^{*}(p)$, the automorphism group of $\Gamma$ is isomorphic, as a permutation group, to the group of permutations of $z(p)$ given by $\{T(a, b) \mid a \in H$ and $b \in z(p)\}$. That this group satisfies all the conclusions of Theorem 3 of [3] is clear.

\section{References}

[1] J.L. Berggren, "An algebraic characterization of finite symmetric tournaments", Bull. Austral. Math. Soc. 6 (1972), 53-59.

[2] Chong-yun Chao, "On groups and graphs", Trans. Amer. Math. Soc. 118 (1965), 488-497.

[3] Chong-yun Chao, "On the classification of symmetric graphs with a prime number of vertices", Trans. Amer. Math. Soc. 158 (1971), $247-256$.

[4] Donald Passman, Permutation groups (W.A. Benjamin, New York, Amsterdam, 1968). 
[5] James Turner, "Point-symmetric graphs with a prime number of points", J. Combinatorial Theory 3 (1967), 136-145.

\author{
Department of Mathematics, \\ Simon Fraser University, \\ Burnaby, \\ British Columbia,
}

Canada. 